\title{
THE DISCRIMINANT MATRIX OF A SEMI-SIMPLE ALGEBRA*
}

\author{
BY \\ C. C. MACDUFFEE
}

1. Introduction. Let $\mathfrak{A}$ be a linear associative algebra with basis $e_{1}, e_{2}$, $\cdots, e_{n}$ over a field $\mathfrak{F}$ in which $n$ has a reciprocal, and let the constants of multiplication be denoted by $c_{i j k}$. Let $t_{1}(x)$ and $t_{2}(x)$ denote, respectively, the first and second traces of $x$. In a recent paper $\dagger$ the writer called the symmetric matrices

$$
T_{1}=\left\|t_{1}\left(e_{r} e_{s}\right)\right\|=\left\|\sum_{i, j} c_{r i j} c_{s j i}\right\|, \quad T_{2}=\left\|t_{2}\left(e_{r} e_{s}\right)\right\|=\left\|\sum_{i, j} c_{i r j} c_{j s i}\right\|
$$

the first and second discriminant matrices of $\mathfrak{A}$ relative to the given basis. The relation of these matrices to the discriminant of an algebraic field was shown, so that the names were justified. It was also shown that under a linear transformation of basis,

$$
e_{i}^{\prime}=\sum a_{i j} e_{j}, A=\left(a_{r s}\right),|A|=a \neq 0,
$$

the matrices are transformed cogrediently, i.e.,

$$
T_{1}^{\prime}=A T_{1} \bar{A}, \quad T_{2}^{\prime}=A T_{2} \bar{A}
$$

where $\bar{A}$ denotes the transpose of $A$. Thus the ranks of $T_{1}$ and $T_{2}$ are invariant, and if $\mathfrak{F}$ is a real field, so are the signatures. Other elementary properties of these matrices were discussed and their occurrence in the literature noted.

In the first part of the present paper the behavior of $T_{1}$ under transformation of basis is used to establish the existence for every algebra with a principal unit of a normal basis of simple form. This normal basis has a cyclic property generalizing that of the familiar basis $1, i, j, k$ for quaternions. By means of this normal basis several new theorems in the theory of semi-simple algebras are obtained, e.g., the fact that $T_{1}$ and $T_{2}$ are identical, and that the first and second characteristic functions are identical.

In the second part of the paper ( $\$ 4$ et seq.) the discriminant matrices of a direct sum, direct product and complete matric algebra are investigated.

2. The normal basis. Let us now assume that $e_{1}$ is a principal unit so that

* Presented to the Society, April 18, 1930; received by the editors in November, 1930.

† Annals of Mathematics, (2), vol. 32, p. 60. 


$$
c_{i 1 j}=c_{1 i j}=\delta_{i j} \text {. }
$$

We denote by $\tau_{r s}$ the elements of $T_{1}$. By means of the associativity conditions we may write $\tau_{r s}$ in the alternative forms*

$$
\tau_{r s}=\sum_{i, k} c_{r i k} c_{s k i}=\sum_{h, k} c_{s r h} c_{h k k}
$$

Then if we set $\sum_{k} c_{h k k}=d_{h}$, we may write $\tau_{r s}=\sum_{h} c_{s+h} d_{h}$. In particular

$$
\begin{aligned}
\tau_{r 1}=\sum_{i, k} c_{r i k} c_{1 k i} & =\sum_{i, k} c_{r i k} \delta_{k i}=\sum_{i} c_{r i i}=d_{r}, \\
\tau_{11} & =\sum_{i} c_{1 i i}=n .
\end{aligned}
$$

Thus $T_{1}$ is of the form

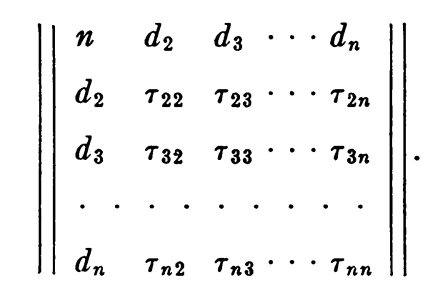

By means of a transformation of matrix

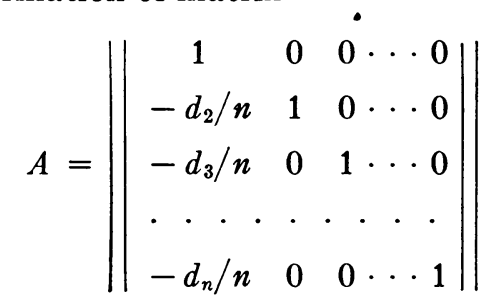

we find that

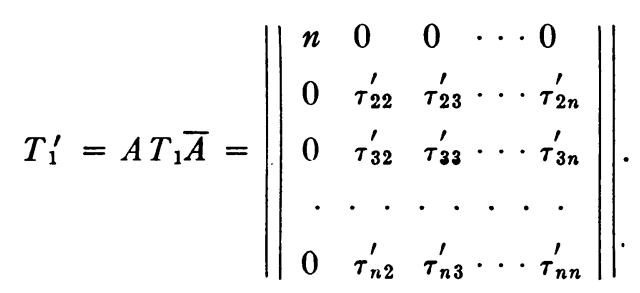

By the above transformation $e_{1}^{\prime}=e_{1}$ is the principal unit. It is now obvious that the symmetric matrix $T_{1}^{\prime}$ can be reduced to a diagonal matrix by transformations in $\mathfrak{F}$ which leave the principal unit $e_{1}$ invariant. We have

\footnotetext{
* MacDuffee, loc. cit., p. 62 (2).
} 
THEOREM 1. If $\mathfrak{X}$ has a principal unit, a basis can be so chosen that the principal unit is $e_{1}$ and

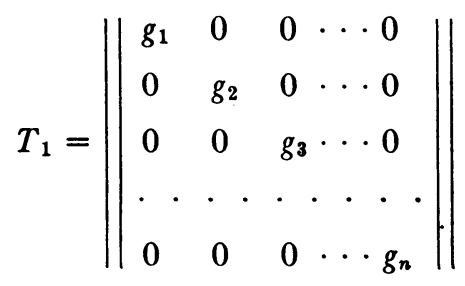

where the $g$ 's are in $\mathfrak{F}$ and $g_{1}=n$.

Such a basis will be called a normal basis for the algebra.

We have seen that when $e_{1}$ is a principal unit, $\tau_{r 1}=d_{r}$, so for a normal basis

$$
d_{1}=n, d_{2}=d_{3}=\cdots=d_{n}=0 \text {. }
$$

Hence* $\tau_{r s}=n c_{s r 1}$ so that

$$
C_{s r 1}=\frac{g_{r}}{n} \delta_{r s} .
$$

In the associativity conditions

$$
\sum_{h} c_{r i h} c_{p h s}=\sum_{h} c_{p r h} c_{h i s}
$$

set $s=1$ and use the above relation. We obtain

$$
\begin{aligned}
\frac{1}{n} \sum_{h} c_{r i h} \delta_{p h} g_{p} & =\frac{1}{n} \sum_{h} c_{p r h} \delta_{h i} g_{i}, \\
g_{p} c_{r i p} & =g_{i} c_{p r i} \quad(i, p, r=1,2, \cdots, n) .
\end{aligned}
$$

THEOREM 2. When a normal basis is taken for an algebra $\mathfrak{A}$ with a principal unit, the constants of multiplication are in the relation (3), the g's being given by (2).

It is interesting to note that for quaternions with the familiar basis 1 , $i, j, k$ where $i^{2}=j^{2}=k^{2}=-1, i j=-j i=k, j k=-k j=i, k i=-i k=j$, the basis is in normal form with $g_{1}=4, g_{2}=g_{3}=g_{4}=-4$. The cyclic property of quaternion multiplication is generalized in the cyclic advance of the subscripts in (3).

3. Some applications. We shall now suppose that $\mathfrak{A}$ is semi-simple with a normal basis $e_{1}, e_{2}, \cdots, e_{n}$. Then each $g_{i}$ in (2) is different from zero.

* Cf. these Transactions, vol. 31 (1929), p. 81, Lemma 7. 
THEOREM 3. In every semi-simple algebra, for every basis, the discriminant matrices $T_{1}$ and $T_{2}$ are identical.

By definition

$$
T_{1}=\left\|\sum_{h, k} c_{r k h} c_{s h k}\right\| .
$$

By means of (3),

$$
\begin{aligned}
T_{1} & =\left\|\sum_{h, k} \frac{g_{k}}{g_{h}} c_{h r k} \frac{g_{h}}{g_{k}} c_{k s h}\right\| \\
& =\left\|\sum_{h, k} c_{h r k} c_{k s h}\right\|=T_{2} .
\end{aligned}
$$

Thus for a normal basis $T_{1}$ and $T_{2}$ are identical. Since they are transformed cogrediently (1), they are identical for all bases.

Henceforth we shall speak of the discriminant matrix of a semi-simple algebra, and denote it by $T$.

THEOREM 4. For every semi-simple algebra and for all bases,

$$
S(x)=T R(x) T^{-1}
$$

where $R(x)$ and $S(x)$ are, respectively, the first and second matrices of $x$, and $T$ is the discriminant matrix.

If $\mathfrak{A}$ has a normal basis, (3) gives

$$
c_{r i s} g_{s}=g_{i} c_{s r i}=g_{r} c_{i s r} .
$$

Hence

$$
S\left(e_{i}\right) T=T R\left(e_{i}\right) .
$$

Multiplying by $x_{i}$ and summing for $i$ gives

$$
S(x) T=T R(x)
$$

where

$$
x=x_{1} e_{1}+x_{2} e_{2}+\cdots+x_{n} e_{n}
$$

is the general number of the algebra. Thus (4) is established for a normal basis.

Under transformation of basis

$$
S(x)=A^{-1} S^{\prime}\left(x^{\prime}\right) A, R(x)=\bar{A} R^{\prime}\left(x^{\prime}\right) \bar{A}^{-1}, *
$$

\footnotetext{
* Dickson, Algebren und ihre Zahlentheorie, p. 38. The $S(x)$ is the transpose of Dickson's $S_{x}$.
} 
and by (1), $T=A^{-1} T^{\prime} \bar{A}^{-1}$. Hence from (4)

$$
\begin{aligned}
A^{-1} S^{\prime}\left(x^{\prime}\right) A & =A^{-1} T^{\prime} \bar{A}^{-1} \bar{A} R^{\prime}\left(x^{\prime}\right) \bar{A}-1 \bar{A} T^{-1} A \\
& =A^{-1} T^{\prime} R^{\prime}\left(x^{\prime}\right) T^{-1} A,
\end{aligned}
$$

so that

$$
S^{\prime}\left(x^{\prime}\right)=T^{\prime} R^{\prime}\left(x^{\prime}\right) T^{\prime-1} .
$$

Thus (4) holds for every basis.

THEOREM 5. For every semi-simple algebra, and for all bases, the first and second characteristic functions are identical.

By definition* the first and second characteristic functions of the general number are

$$
\begin{aligned}
& C_{1}(\omega)=|R(x)-\omega I|, \\
& C_{2}(\omega)=|S(x)-\omega I| .
\end{aligned}
$$

The theorem follows immediately from (4).

4. The discriminant matrix of a direct sum. An algebra $\mathfrak{A}$ is the direct sum $\mathfrak{B} \oplus \mathfrak{C}$ of two algebras $\mathfrak{B}$ and $\mathfrak{C}$ if $\mathfrak{A}=\mathfrak{B}+\mathfrak{C}, \mathfrak{B} \mathfrak{C}=\mathfrak{C} \mathfrak{B}=0, \mathfrak{B} \wedge \mathfrak{C}=0$. It is known that every semi-simple algebra is the direct sum of simple algebras, and the components are unique except for order.

The direct sum of two matrices $M_{1}$ and $M_{2}$ is the matrix

$$
\left\|\begin{array}{ll}
M_{1} & O \\
O & M_{2}
\end{array}\right\|
$$

where the $O$ 's stand for rectangular blocks of 0 's. Let $T(\mathfrak{A})$ denote the discriminant matrix of $\mathfrak{A}$.

THEOREM 6. If $\mathfrak{A}=\mathfrak{B} \oplus \mathfrak{E}$, a basis for $\mathfrak{A}$ may be so chosen that

$$
T(\mathfrak{A})=T(\mathfrak{B}) \oplus T(\mathfrak{S}) .
$$

We choose the basis numbers $e_{1}, e_{2}, \cdots, e_{n}$ of $\mathscr{U}$ so that $e_{1}, e_{2}, \cdots, e_{h}$ form a basis for $\mathfrak{B}$ and $e_{h+1}, \cdots, e_{n}$ a basis for $\mathfrak{E}$. Then $e_{r} e_{s}=0$ unless $r \leqq h, s \leqq h$ or $r>h, s>h$. Since $T(\mathfrak{A})=\left\|t\left(e_{r} e_{s}\right)\right\|$, obviously

$$
T(\mathfrak{A})=\left\|\begin{array}{cc}
T(\mathfrak{B}) & 0 \\
0 & T(\mathfrak{S})
\end{array}\right\| .
$$

COROLlaRy 6. If $\mathfrak{F}$ is a real field, and $\mathfrak{A}=\mathfrak{B} \oplus \mathfrak{C}$, then the signature of $T(\mathfrak{A})$ is the sum of the signatures of $T(\mathscr{B})$ and $T(\mathfrak{S})$.

* Dickson, ibid., p. 37. 
5. The discriminant matrix of a direct product. An algebra $\mathfrak{A}$ is the direct product $\mathfrak{B} \times \mathfrak{C}$ of two algebras $\mathfrak{B}$ and $\mathfrak{C}$ if $\mathfrak{A}=\mathfrak{B} \mathfrak{C}$, the order of $\mathfrak{A}$ is the product of the orders of $\mathfrak{B}$ and $\mathfrak{S}$, and if every number of $\mathfrak{B}$ is commutative with 'every number of $\mathfrak{c}$.

If $P=\left(p_{r s}\right)$ and $Q=\left(q_{r s}\right)$ are two square matrices, the direct product* $P \times Q$ is defined to be the matrix

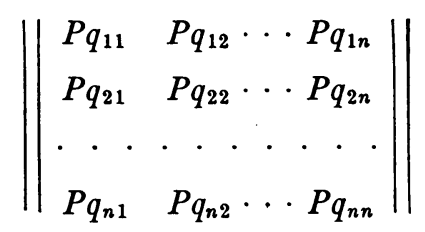

where $P q_{i j}$ stands for the block of elements

$$
\begin{aligned}
& p_{11} q_{i j} \quad p_{12} q_{i j} \cdots p_{1 n} q_{i j} \\
& p_{21} q_{i j} \quad p_{22} q_{i j} \cdots p_{2 n} q_{i j} \\
& p_{n 1} q_{i j} \quad p_{n 2} q_{i j} \cdots p_{n n} q_{i j} .
\end{aligned}
$$

TheOREM 7. If $\mathfrak{A}=\mathfrak{B} \times \mathfrak{C}$, a basis for $\mathfrak{A}$ can be so chosen that

$$
T(\mathfrak{A})=T(\mathfrak{B}) \times T(\mathfrak{S}) .
$$

Suppose that $\mathfrak{B}$ has the basis $e_{1}, e_{2}, \cdots, e_{0}$ and the constants of multiplication $b_{i j k}$, while $\mathbb{C}$ has the basis $f_{1}, f_{2}, \cdots, f_{h}$ and the constants of multiplication $c_{i j k}$. Then the numbers $e_{i_{1}} f_{i_{2}}$ form a basis for $\mathfrak{A}$, and

$$
\begin{aligned}
e_{i_{1}} f_{i_{2}} e_{j_{1}} f_{i_{2}} & =e_{i_{1}} e_{j_{1}} f_{i_{2}} f_{j_{2}} \\
& =\sum_{\substack{k_{1}=1, \cdots, g \\
k_{2} \neq 1, \cdots, h}} b_{i_{1} j_{1} k_{1}} c_{i_{2} j_{2} k_{2}} e_{k_{1}} f_{k_{2}} .
\end{aligned}
$$

Let us denote the basis numbers $e_{i_{1}} f_{i_{2}}$ of $\mathfrak{A}$ by the symbols $E_{i}$ and order them so that

$$
i-1=g\left(i_{2}-1\right)+i_{1}-1,0 \leqq i_{1}-1<g .
$$

Evidently $i_{1}$ and $i_{2}$ determine $i$ uniquely and conversely. Then (5) may be written

* A. Hurwitz, Mathematische Annalen, vol. 45, p. 381.

C. Stephanos, Journal de Mathématiques, (5), vol. 6, p. 73. 


$$
E_{i} E_{j}=\sum_{k=1}^{n} D_{i j k} E_{k}
$$

where $D_{i j k}=b_{i_{1} j_{1} k_{1}} c_{i_{2} j_{2} k_{2}}$, the $j_{1}, j_{2}, k_{1}, k_{2}$ being determined from $j$ and $k$ by relations similar to (6).

It now follows that

$$
\begin{aligned}
T(\mathfrak{A}) & =\left\|\sum_{p, q=1}^{g h} D_{r p q} D_{s q p}\right\| \\
& =\left\|\sum_{p_{1}, q_{1}, p_{2}, q_{2}} b_{r_{1} p_{1} q_{1}} c_{r_{2} p_{2} q_{2}} b_{s_{1} q_{1} p_{1}} c_{s_{2} q_{2} p_{2}}\right\| \\
& =\left\|\sum_{p_{1}, q_{1}} b_{r_{1} p_{1} q_{1}} b_{s_{1} q_{1} p_{1}} \sum_{p_{2}, q_{2}} c_{r_{2} p_{2} q_{2}} c_{s_{2} q_{2} p_{2}}\right\| \\
& =T(\mathfrak{B}) \times T(\mathfrak{S}) .
\end{aligned}
$$

CoROllary 7. If $\mathfrak{F}$ is a real field, and $\mathfrak{A}=\mathfrak{B} \times \mathfrak{(}$, the signature of $T(\mathfrak{A})$ is equal to the product of the signatures of $T(\mathfrak{B})$ and $T(\mathfrak{S})$.

As in Theorem 1 bases for $\mathfrak{B}$ and $\mathbb{E}$ may be so chosen that $T(\mathfrak{B})$ and $T(\mathfrak{C})$ are diagonal matrices. It is evident that $T(\mathfrak{A})$ is now a diagonal matrix whose diagonal elements are the products of the diagonal elements of $T(\mathfrak{B})$ by the diagonal elements of $T(\mathbb{S})$. Let $p_{1}, p_{2}$ and $p$ denote, respectively, the number of positive terms in the main diagonals of $T(\mathfrak{B}), T(\mathfrak{C})$ and $T(\mathfrak{A})$. Similarly let $n_{1}, n_{2}$ and $n$ denote the number of negative terms. Then evidently

$$
\begin{aligned}
& p=p_{1} p_{2}+n_{1} n_{2}, \quad n=p_{1} n_{2}+p_{2} n_{1}, \\
& p-n=\left(p_{1}-n_{1}\right)\left(p_{2}-n_{2}\right) .
\end{aligned}
$$

6. The discriminant matrix of a complete matric algebra. If $\mathfrak{A}$ is a complete matric algebra of order $n^{2}$, basal numbers $e_{i j}$ can be so chosen that

$$
e_{i j} e_{k l}=\delta_{j k} e_{i l}
$$

where $\delta_{j k}$ is Kronecker's delta. We shall arrange the $e_{i j}$ in the order $e_{11}, e_{22}$, $\cdots, e_{n n}$ followed by $e_{i j}, e_{j i}, j>i ; i=1,2, \cdots ; j=i+1, \cdots$. If we denote $T(\mathfrak{A})$ by $\left(\tau_{r s}\right)$, then

$$
\begin{aligned}
\tau_{r s} & =t\left(e_{r_{1} r_{2}} e_{{\theta_{1} s_{2}}_{2}}\right)=t\left(\delta_{r_{2} s_{1}} e_{r_{1} s_{2}}\right)=n \delta_{r_{2} s_{1}} t\left(e_{r_{1} s_{2}}\right) \\
& =n \delta_{r_{2} s_{1}} \delta_{r_{1 s_{2}}} .
\end{aligned}
$$

Thus $T(\mathfrak{A})$ is in the form 
(7)

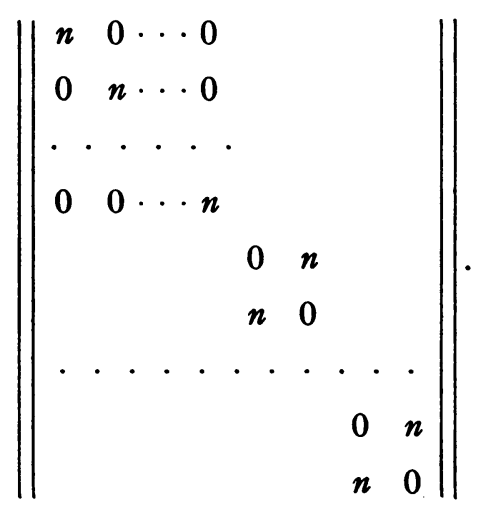

TheOREM 8. If $\mathfrak{A}$ is a complete matric algebra, basal numbers may be so chosen that $T(\mathscr{A})$ is of the form (7).

COROLLARY 8. If $\mathfrak{F}$ is a real field and $\mathfrak{A}$ is a complete matric algebra of order $n^{2}$, the signature of $T(\mathfrak{X})$ is $n$.

The OHo State University, Colomats, OHo 